\title{
Upcoming market catalysts in Q3 2016
}

Important market catalysts expected in the third quarter of 2016 include an FDA approval decision for the universal factor $\mathrm{X}$ a inhibitor antidote, andexanet alfa (developed by Portola Pharmaceuticals), top-line results from the Phase III programme of guselkumab (developed by MorphoSys and Janssen Biotech) for psoriasis, and top-line Phase II results for GR-MD-02 (developed by Galectin) to treat advanced fibrosis patients with nonalcoholic steatohepatitis (NASH).

Portola is seeking FDA approval for andexanet alfa, an anticoagulant reversal agent. Portola submitted a biologics license application (BLA) under an accelerated approval pathway in December 2015 and was granted priority review. A decision is expected by the Prescription Drug User Fee Act (PDUFA) action date of 17 August 2016. Previously, the FDA granted breakthrough therapy and orphan designations to andexanet alfa. The BLA is supported by the Phase III ANNEXA studies, which evaluated the safety and efficacy of andexanet alfa in reversing the anticoagulant activity of the factor $\mathrm{Xa}$ inhibitors rivaroxaban and apixaban in healthy volunteers. The studies showed that andexanet alfa reversed anticoagulant activity within minutes of administration and the effect was sustained throughout a 2-hour infusion. An approval for andexanet alfa could lead to increased use of direct factor $\mathrm{Xa}$ inhibitor anticoagulants among physicians who have been concerned about the lack of an antidote in case of serious emergency bleeding. Idarucizumab (Praxbind; Boehringer Ingelheim), a reversal agent for the direct thrombin inhibitor dabigatran (Pradaxa; Boehringer Ingelheim), was approved by the FDA in October 2015.

Top-line data readouts from the Phase III programme of guselkumab for psoriasis are expected in mid-2016. Guselkumab is a fully human monoclonal antibody ( $\mathrm{mAb}$ ) that binds to the p19 subunit of interleukin-23 (IL-23), thereby potentially targeting IL-23 signalling more selectively than the approved psoriasis therapy ustekinumab (Stelara; Janssen Biotech), a mAb that binds to the $\mathrm{p} 40$ subunit shared by IL-23 and IL-12. Guselkumab is currently in three Phase III studies (known as VOYAGE 1, VOYAGE 2 and NAVIGATE) and being tested at the $100 \mathrm{mg}$ dose, which has shown promising results in Phase Il testing compared with placebo as well as with adalimumab (Humira; AbbVie), a tumour necrosis factor (TNF)-specific $\mathrm{mAb}$ that is widely used to treat psoriasis. The primary end point of a physician's global assessment (PGA) score of either 0 or 1 at week 16 was achieved in $86 \%$ of patients receiving guselkumab versus $58 \%$ receiving adalimumab $(P<0.05)$. Additionally, at week $16,79 \%$ and $62 \%$ of patients on guselkumab achieved $75 \%$ reductions in the Psoriasis Area and Severity Index (PASI) score and $90 \%$ reductions in the PASI score, respectively. The Phase III studies will look to replicate these results and, in the NAVIGATE study in particular, a direct comparison of guselkumab with ustekinumab will be available. If the results of the Phase III data programme are positive, MorphoSys and Janssen Biotech anticipate a BLA submission to the FDA before the end of 2016 .

Finally, top-line results from the Phase II NASH-FX study of GR-MD-02, a carbohydrate-based galectin-3 inhibitor, are expected by the end of September 2016. NASH-FX completed enrolment of 30 liver biopsy-confirmed NASH patients with advanced fibrosis (stage 3) in May 2016. Patients were randomized to placebo or $8 \mathrm{mg}$ per kg GR-MD-02 on a 1:1 ratio. The primary objective of the study is to determine the difference between placebo and GR-MD-02 in the baseline-adjusted mean change in liver fibrosis as measured by corrected T1 (cT1). In a previous Phase I study, reductions in the extent of liver fibrosis were observed, but a statistically significant reduction was only observed at the $8 \mathrm{mg}$ per $\mathrm{kg}$ dosing level, the same dose being tested in the current Phase II study. Another Phase II trial, NASH-CX, is currently ongoing. This study will focus on patients with cirrhosis. Together, the results of these trials should guide Galectin in the formation of their Phase III programme for $\mathrm{NASH}$, an indication with no FDA-approved therapies at present.

\footnotetext{
Tyler Ranj is at Informa, 3655 Nobel Drive, San Diego, California 92122, USA. TRanj@sagientresearch.com doi: 10.1038/nrd.2016.119 Published online 10 Jun 2016

The author declares no competing interests.
} 